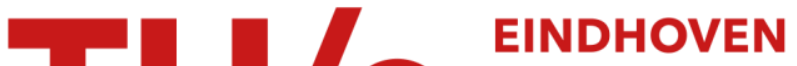

\section{$\mathrm{Ab}$ initio theory of charge-carrier conduction in ultrapure organic crystals}

\section{Citation for published version (APA):}

Hannewald, K., \& Bobbert, P. A. (2004). Ab initio theory of charge-carrier conduction in ultrapure organic crystals. Applied Physics Letters, 85(9), 1535-1537. https://doi.org/10.1063/1.1776335

DOI:

10.1063/1.1776335

Document status and date:

Published: 01/01/2004

\section{Document Version:}

Publisher's PDF, also known as Version of Record (includes final page, issue and volume numbers)

\section{Please check the document version of this publication:}

- A submitted manuscript is the version of the article upon submission and before peer-review. There can be important differences between the submitted version and the official published version of record. People interested in the research are advised to contact the author for the final version of the publication, or visit the $\mathrm{DOI}$ to the publisher's website.

- The final author version and the galley proof are versions of the publication after peer review.

- The final published version features the final layout of the paper including the volume, issue and page numbers.

Link to publication

\section{General rights}

Copyright and moral rights for the publications made accessible in the public portal are retained by the authors and/or other copyright owners and it is a condition of accessing publications that users recognise and abide by the legal requirements associated with these rights.

- Users may download and print one copy of any publication from the public portal for the purpose of private study or research.

- You may not further distribute the material or use it for any profit-making activity or commercial gain

- You may freely distribute the URL identifying the publication in the public portal.

If the publication is distributed under the terms of Article 25fa of the Dutch Copyright Act, indicated by the "Taverne" license above, please follow below link for the End User Agreement:

www.tue.nl/taverne

Take down policy

If you believe that this document breaches copyright please contact us at:

openaccess@tue.nl

providing details and we will investigate your claim. 


\title{
$A b$ initio theory of charge-carrier conduction in ultrapure organic crystals
}

\author{
K. Hannewald ${ }^{\text {a) }}$ and P. A. Bobbert \\ Group Polymer Physics, Eindhoven Polymer Laboratories, Technische Universiteit Eindhoven, \\ P. O. Box 513, 5600 MB Eindhoven, The Netherlands
}

(Received 10 March 2004; accepted 4 June 2004)

\begin{abstract}
We present an ab initio description of charge-carrier mobilities in organic molecular crystals of high purity. Our approach is based on Holstein's original concept of small-polaron bands but generalized with respect to the inclusion of nonlocal electron-phonon coupling. By means of an explicit expression for the mobilities as a function of temperature in combination with ab initio calculations of the material parameters, we demonstrate the predictive power of our theory by applying it to naphthalene. The results show a good qualitative agreement with experiment and provide insight into the difference between electron and hole mobilities as well as their peculiar algebraic and anisotropic temperature dependencies. (C) 2004 American Institute of Physics.
\end{abstract}

[DOI: $10.1063 / 1.1776335]$

Organic semiconductors are promising materials for low-cost and easy-to-process (opto)electronic devices. ${ }^{1-4} \mathrm{Be}-$ sides $\pi$-conjugated polymers, an important material class is organic molecular crystals of high purity. Due to their high degree of structural order, such crystals are ideal candidates for the investigation of the intrinsic charge-carrier transport phenomena in organic solids. Thus, many interesting fundamental studies have been performed in recent years and new results emerge at a rapid pace. ${ }^{5-9}$ A particularly important topic and "still a great challenge for theoreticians"10 is the understanding of the charge-carrier mobilities in these crystals.

In comparison to covalently bonded inorganic semiconductors, organic molecular crystals exhibit weak intermolecular bonds and, hence, narrower electron bands and stronger electron-lattice interaction. As a result, the dressing of the charge carriers by phonon clouds, i.e., polaron formation, becomes quite important. Pioneering theoretical work in this area was done by Holstein, ${ }^{11}$ who introduced the concept of small-polaron bands for a local (on-site) electron-phonon coupling. The main prediction of his theory is that the mobilities initially decrease with rising temperature $T$ due to bandwidth narrowing but increase again for higher $T$ due to phonon-assisted hopping. Two decades later, the interplay between metallic (bandlike) conduction and activated (hopping) transport in organic crystals was seen for the electrons in naphthalene but not for the holes. ${ }^{12-14}$ Later, Kenkre et al. were able to fit the measured electron mobilities reasonably well to Holstein's model, assuming directionally dependent local-coupling constants. ${ }^{15}$ Despite the success of such a fitting procedure, the lack of a first-principles description of charge-carrier mobilities in organic crystals has left several fundamental questions unanswered. This concerns, in particular, the different behavior of electrons and holes, the microscopic origin of the crystallographic anisotropy in the $T$ dependence, and the influence of nonlocal (Peierls-type) couplings such as present in the Su-Schrieffer-Heeger model. ${ }^{16-18}$

In this letter, we address the above-noted questions by presenting a first-principles approach to charge-carrier mo-

\footnotetext{
a)Electronic mail: k.k.hannewald@tue.nl
}

bilities in organic crystals. We consider a mixed HolsteinPeierls model for the interaction between electrons (holes) and phonons. In a tight-binding description, this corresponds to a Hamiltonian of the form $H=\sum_{m n} \varepsilon_{m n} a_{m}^{\dagger} a_{n}+\Sigma_{\mathbf{Q}} \hbar \omega_{\mathbf{Q}}\left(b_{\mathbf{Q}}^{\dagger} b_{\mathbf{Q}}\right.$ $\left.+\frac{1}{2}\right)+\sum_{\mathrm{mn} \mathbf{Q}} \hbar \omega_{\mathbf{Q}} g_{\mathbf{Q} m n} a_{m}^{\dagger} a_{n}\left(b_{\mathbf{Q}}^{\dagger}+b_{-\mathbf{Q}}\right)$. Here, the operators $a_{m}^{(\dagger)}$ and $b_{\mathbf{Q}}^{(\dagger)}:=b_{\mathbf{q} \lambda}^{(\dagger)}$ annihilate (create) an electron at equivalent lattice sites $\mathbf{R}$ with energy $\varepsilon_{m m}$ and a phonon in the mode $\lambda$ with wave vector $\mathbf{q}$ and frequency $\omega_{\lambda \mathbf{q}}=: \omega_{\mathbf{Q}}$, respectively. The strength of the electron-lattice interaction is determined by the dimensionless quantities $g_{\mathbf{Q} m n}$ that describe both the local coupling to the on-site energies $\varepsilon_{m m}$ (Holstein model) and the nonlocal coupling to the transfer integrals $\varepsilon_{m n}$ ( $m$ $\neq n$, Peierls model).

In thermal equilibrium, the mobility $\mu_{\alpha}$ in direction $\mathbf{e}_{\alpha}$ can be calculated by means of the Kubo formula for electrical conductivity. This basically amounts to evaluating a current-current correlation function where, in general, the current $\mathbf{j}:=\mathbf{j}^{(\mathrm{I})}+\mathbf{j}^{(\mathrm{II})}$ is composed of a purely electronic term $\mathbf{j}^{(\mathrm{I})}=\left(e_{0} / i \hbar\right) \Sigma_{m n}\left(\mathbf{R}_{m}-\mathbf{R}_{n}\right) \varepsilon_{m n} a_{m}^{\dagger} a_{n}$ and a phonon-assisted current $\mathbf{j}^{(\mathrm{II})}=\left(e_{0} / i \hbar\right) \Sigma_{m n \mathbf{Q}}\left(\mathbf{R}_{m}-\mathbf{R}_{n}\right) \hbar \omega_{\mathbf{Q}} g_{\mathbf{Q} m n}\left(b_{\mathbf{Q}}^{\dagger}+b_{-\mathbf{Q}}\right) a_{m}^{\dagger} a_{n}$. We stress that the current $\mathbf{j}^{(\mathrm{II})}$ originates solely from the nonlocal electron-phonon coupling. In a recent article, ${ }^{19}$ we have performed an analytical evaluation of the Kubo formula using the method of canonical transformation which allowed us to incorporate the electron-phonon interaction in a nonperturbative manner, in accordance with the underlying physical picture of small-polaron bands. Here, we give only the results for the mobilities in the case of dispersionless optical phonons $\left(\omega_{\mathbf{Q}} \rightarrow \omega_{\lambda}\right)$.

For the mobility $\mu_{\alpha}^{(I)}$ due to the current $\mathbf{j}^{(\mathrm{I})}$, we obtain

$$
\begin{aligned}
\mu_{\alpha}^{(I)}= & \frac{e_{0}}{2 k_{B} T \hbar^{2}} \sum_{n \neq m}\left(R_{\alpha m}-R_{\alpha n}\right)^{2} \int_{-\infty}^{+\infty} d t \\
& \times\left[\varepsilon_{m n} e^{-\Sigma_{\lambda} G_{\lambda}\left[1+2 N_{\lambda}-\Phi_{\lambda}(t)\right]}\right]^{2} e^{-\Gamma^{2} t^{2}},
\end{aligned}
$$

where $N_{\lambda}=\left(e^{\hbar \omega_{\lambda} / k_{B} T}-1\right)^{-1}$ denotes the phonon occupation number and $\Gamma$ is a phenomenological broadening parameter that accounts for scattering processes beyond our model, e.g., due to defects. Those terms in the exponent of Eq. (1) that contain $\Phi_{\lambda}(t)=\left(1+N_{\lambda}\right) e^{-i \omega_{\lambda} t}+N_{\lambda} e^{i \omega_{\lambda} t}$ describe incoherent scattering events involving actual changes in phonon 


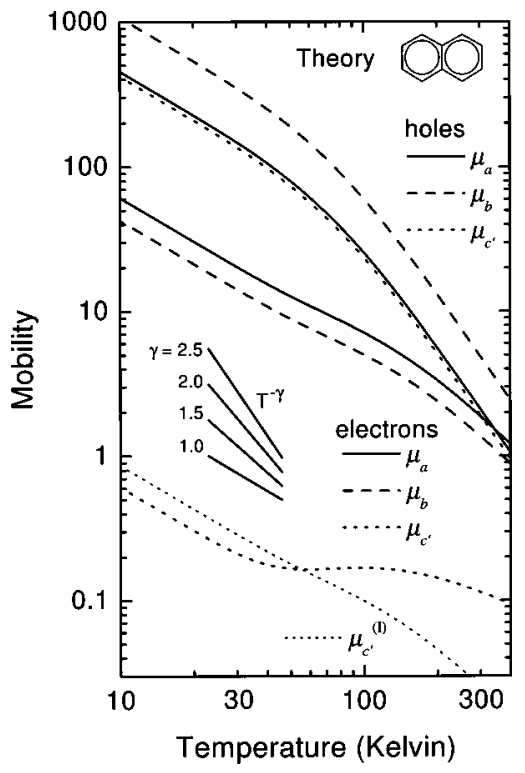

FIG. 1. Calculated charge-carrier mobilities (in arbitary units, see Refs. 22 and 23) vs temperature $T$ for naphthalene crystals, as obtained from Eqs. (1) and (2). As a guide to the eye, several power laws $\mu_{\alpha} \propto T^{-\gamma}$ are also depicted.

numbers (hopping) whereas the remaining terms account for purely coherent scattering processes (bandwidth narrowing). While Eq. (1) is qualitatively similar to the Holstein-model mobilities obtained by Kenkre et al., ${ }^{15}$ an important quantitative difference is that the exponent in our formula (1) is not only governed by the local coupling but by effective coupling constants $G_{\lambda}=\left(g_{\lambda m m}\right)^{2}+\frac{1}{2} \sum_{k \neq m}\left(g_{\lambda m k}\right)^{2}$ that are composed of both the local and nonlocal ones.

In order to obtain the total mobilities $\mu_{\alpha}$ within our Holstein-Peierls model, we have to include also the contributions from the phonon-assisted current $\mathbf{j}^{\text {(II) }}$. This results in an expression that is identical in form to Eq. (1) but with the replacement

$$
\left(\varepsilon_{m n}\right)^{2} \rightarrow\left(\varepsilon_{m n}-\Delta_{m n}\right)^{2}+\frac{1}{2} \sum_{\lambda}\left(\hbar \omega_{\lambda} g_{\lambda m n}\right)^{2} \Phi_{\lambda}(t),
$$

where $\quad \Delta_{m n}=\frac{1}{2} \Sigma_{\lambda} \hbar \omega_{\lambda}\left[g_{\lambda m n}\left(g_{\lambda m m}+g_{\lambda n n}\right)+\frac{1}{2} \sum_{k \neq m, n} g_{\lambda m k} g_{\lambda k n}\right]$. An essential qualitatively new feature compared to Eq. (1) is that the total mobilities $\mu_{\alpha}$ can account for an anisotropic $T$ dependence due to the extra term containing $\Phi_{\lambda}(t)$ in Eq. (2), which describes additional phonon-assisted hopping processes that are absent in local-coupling theories and reflect an inherently transport-promoting effect solely caused by the nonlocal coupling.

We proceed by applying our mobility theory to naphthalene, which crystallizes in a monoclinic structure and exhibits a herringbone stacking with two equivalent molecules per unit cell. In order to obtain the material-specific parameters $\mathbf{R}_{m}, \varepsilon_{m n}, g_{\lambda m n}$, and $\omega_{\lambda}$ we use a three-step strategy described in more detail in Ref. 20.

First, we determine the equilibrium structure of the crystal by means of state-of-the-art DFT-LDA calculations using the $a b$ initio total-energy and molecular dynamics program VASP. ${ }^{21}$ For the resulting geometry $(a=7.68 \AA, b=5.76 \AA, c$ $\left.=8.35 \AA, \beta=125.7^{\circ}\right)$, the intermolecular optical-phonon energies $\left(\hbar \omega_{\lambda}=10.7,14.2,17.4 \mathrm{meV}\right)$ and eigenvectors are obtained within the rigid-molecule approximation. ${ }^{22}$ Second, we obtain the values $\varepsilon_{m n}$ from a fit of the ground-state $a b$ Downloaded 08 Nov 2007 to 131.155.108.71. Redistribution subject

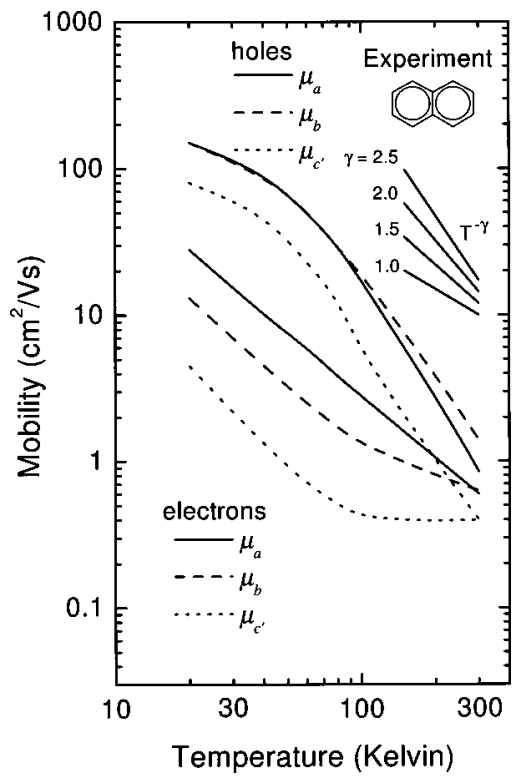

FIG. 2. Experimental charge-carrier mobilities vs temperature $T$ for naphthalene crystals, as extracted from Ref. 14.

initio highest occupied molecular orbital (HOMO) and lowest unoccupied molecular orbital (LUMO) energy bands to a tight-binding model, including the six most important transfer integrals. Third, the coupling constants $g_{\lambda m n}$ are obtained by rotating the molecules according to the phonon eigenvectors and fitting the resulting ab initio band structure to the Holstein-Peierls Hamiltonian. A compilation of all calculated material parameters can be found in Ref. 20. Finally, we have chosen a small line broadening of $\hbar \Gamma=0.1 \mathrm{meV}$, corresponding to the case of ultrapure crystals. ${ }^{23}$

In Fig. 1, we present the calculated electron and hole mobilities in naphthalene crystals as a function of temperature $T$ for the $a, b$, and $c^{\prime}$ directions, with $c^{\prime}$ being perpendicular to the $a b$ plane of the molecular layers. In Fig. 2, the corresponding experimental mobility data are replotted from the work of Karl. ${ }^{14}$

We make three important observations. First, the calculated hole mobilities are generally larger than the corresponding electron mobilities. Only at $T \approx 300 \mathrm{~K}$ do we observe comparable in-plane mobilities for electrons and holes. This general trend is in good agreement with the experimental data, apart from the overestimated theoretical spread between electrons and holes for $\mu_{c^{\prime}}$, which might be related to the difficulties of LDA in describing van der Waals bonds. We stress that the lower electron mobilties cannot be simply understood from the electronic transfer integrals but that they are mainly due to their stronger coupling to the phonons [LUMO: $\Sigma_{\lambda} G_{\lambda}=(1.52)^{2}$, HOMO: $\left.\Sigma_{\lambda} G_{\lambda}=(0.92)^{2}\right]$. We note also that our calculated effective coupling constant of 1.52 for the LUMO is comparable in value to the directionally dependent fitted coupling values of $1.62,1.83$, and $1.88 \mathrm{ob}-$ tained by Kenkre et al. ${ }^{15}$

Second, while the hole mobilities in the different directions decrease very similarly with $T$, the electron mobilities exhibit a pronounced anisotropic $T$ dependence. In particular, we find a metallic (bandlike) behavior within the $a b$ plane and a slightly activated (hopping) transport in the $c^{\prime}$ direction. Again, these findings from our $a b$ initio calculations are in good agreement with the experiment, which shows such nearly $T$-independent mobility around $T \approx 150 \mathrm{~K}$ exclusively to AlP license or copyright, see http://apl.aip.org/apl/copyright.jsp 
in the $c^{\prime}$ direction. As discussed earlier this pronounced anisotropy is solely due to the phonon-assisted currents $\mathbf{j}^{\text {(II) }}$ and, in fact, the peculiar flattering seen for $\mu_{c^{\prime}}$ is not obtained at all for $\mu_{c^{\prime}}^{(\mathrm{I})}$ alone. This clearly demonstrates that anisotropy effects in the $T$ dependence can be explained in a natural way by the inclusion of nonlocal electron-phonon coupling, and do not require the use of additional concepts such as directionally dependent local couplings ${ }^{15}$ or correlated successive hops of strongly localized charge carriers. ${ }^{24}$

Third, our calculated hole mobilities obey at elevated $T$ the power law $\mu_{\alpha} \propto T^{-2.5}$. This agrees well with Karl's experiments where $\mu_{\alpha} \propto T^{-2.9}, T^{-2.5}, T^{-2.8}$. This peculiar powerlaw dependence, which has been poorly understood so far, can be straightforwardly understood from our theory. In the limit of small broadening $\hbar \Gamma$ and for $\mu_{\alpha} \approx \mu_{\alpha}^{\text {(I) }}$ (here fulfilled for the holes), a spectral analysis of Eq. (1) shows that the dominant electron-phonon scattering processes are those that allow only energy exchange within each phonon mode but not between different modes, and the mobility can be expressed in terms of modified Bessel functions,

$$
\mu_{\alpha}^{(\mathrm{I})} \propto \frac{1}{\Gamma} \frac{1}{k_{B} T}\left(\Pi_{\lambda} e^{-2 G_{\lambda}\left(1+2 N_{\lambda}\right)} I_{0}\left(z_{\lambda}\right)\right),
$$

where $z_{\lambda}=4 G_{\lambda} \sqrt{N_{\lambda}\left(1+N_{\lambda}\right)}$. The limit of high $T$ is obtained using $I_{0}\left(z_{\lambda} \rightarrow \infty\right) \approx e^{z_{\lambda}} / \sqrt{2 \pi z_{\lambda}}$ and we find $\mu_{\alpha}^{(\mathrm{I})}$ $\propto T^{-1}\left(\Pi_{\lambda=1}^{3} T^{-0.5}\right)=T^{-2.5}$, which indicates that the exponent is actually a measure for the number of relevant phonon modes per molecule. For the electrons, the situation is more complicated due to a subtle interplay between the (here significant) contributions of $\mathbf{j}^{\text {(II) }}$ and the larger coupling values. This prohibits an easy analytical evaluation but, by looking at our calculated in-plane electron mobilities in Fig. 1, we find at high $T$ an approximate $T^{-1.5}$ dependence, again in close agreement with the experiment. The disagreement at low $T$ between theory $\left(\mu_{\alpha} \propto T^{-1}\right)$ and experiment $\left(\mu_{\alpha}\right.$ $\left.\propto T^{-1.4}, T^{-1.5}, T^{-1.6}\right)$ might be due to the neglect of acoustic phonons. Following our above discussion, they may be considered as effectively one dispersive phonon branch that could give rise to the missing factor $T^{-0.5}$.

In summary, we have presented a theoretical description of charge-carrier mobilities in ultrapure organic crystals based on a mixed Holstein-Peierls model. The predictive power of our theory has been demonstrated by ab initio studies for naphthalene crystals where our results show good qualitative agreement with experiment and provide microscopic insight into several hitherto poorly understood phenomena. Since our theory may also be applied to other weakly bonded materials with strong electron-phonon coupling, it may become an important contribution to the understanding of the charge-carrier transport in these materials, too.

The authors wish to thank D. Emin, S. Mazumdar, J. Pflaum, S. Stafström, and J. van den Brink for valuable discussions. Financial support by the Dutch Foundation for Fundamental Research on Matter (FOM) is acknowledged.

${ }^{1}$ N. S. Sariciftci, L. Smilowitz, A. J. Heeger, and F. Wudl, Science 258, 1474 (1992).

${ }^{2}$ N. Tessler, G. J. Denton, and R. H. Friend, Nature (London) 382, 695 (1996).

${ }^{3}$ R. H. Friend, R. W. Gymer, A. B. Holmes, J. H. Burroughes, R. N. Marks, C. Taliani, D. D. C. Bradley, D. A. Dos Santos, J. L. Brédas, M. Logdlund, and W. R. Salaneck, Nature (London) 397, 121 (1999).

${ }^{4}$ N. Stutzmann, R. H. Friend, and H. Sirringhaus, Science 299, 1881 (2003).

${ }^{5}$ V. Coropceanu, M. Malagoli, D. A. de Silva Filho, N. E. Gruhn, T. G. Bill, and J. L. Brédas, Phys. Rev. Lett. 89, 275503 (2002).

${ }^{6}$ S. Fratini and S. Ciuchi, Phys. Rev. Lett. 91, 256403 (2003).

${ }^{7}$ V. Podzorov, S. E. Sysoev, E. Loginova, V. M. Pudalov, and M. E. Gershenson, Appl. Phys. Lett. 83, 3504 (2003).

${ }^{8}$ R. W. I. de Boer, T. M. Klapwijk, and A. F. Murpurgo, Appl. Phys. Lett. 83, 4345 (2003).

${ }^{9}$ V. Y. Butko, X Chi, D. V. Lang, and A. P. Ramirez, Appl. Phys. Lett. 83, 4773 (2003).

${ }^{10}$ N. Karl, Synth. Met. 133/134, 649 (2003).

${ }^{11}$ T. Holstein, Ann. Phys. (N.Y.) 8, 343 (1959).

${ }^{12}$ L. B. Schein, C. B. Duke, and A. R. McGhie, Phys. Rev. Lett. 40, 197 (1978).

${ }^{13}$ W. Warta and N. Karl, Phys. Rev. B 32, 1172 (1985).

${ }^{14}$ N. Karl, in Landolt-Börnstein: Numerical Data and Functional Relationship in Science and Technology, edited by K.-H. Hellwege and O. Madelung (Springer, Berlin, 1985), Group III, Vol. 17, Pt. i, p. 106.

${ }^{15}$ V. M. Kenkre, J. D. Andersen, D. H. Dunlap, and C. B. Duke, Phys. Rev. Lett. 62, 1165 (1989).

${ }^{16}$ W. P. Su, J. R. Schrieffer, and A. J. Heeger, Phys. Rev. Lett. 42, 1698 (1979).

${ }^{17}$ H. Sumi, J. Chem. Phys. 70, 3775 (1979).

${ }^{18}$ R. W. Munn and R. Silbey, J. Chem. Phys. 83, 1854 (1985).

${ }^{19}$ K. Hannewald and P. A. Bobbert, Phys. Rev. B 69, 075212 (2004).

${ }^{20}$ K. Hannewald, V. M. Stojanovic, J. M. T. Schellekens, P. A. Bobbert, G. Kresse, and J. Hafner, Phys. Rev. B 69, 075211 (2004).

${ }^{21}$ G. Kresse and J. Hafner, Phys. Rev. B 47, 558 (1993); 49, 14251 (1994); G. Kresse and J. Furthmüller, ibid. 54, 11169 (1996).

${ }^{22}$ In principle, our approach does also allow the inclusion of intramolecular phonons. However, due to their higher frequencies (Ref. 5), i.e., lower occupations, we expect them to have little influence on the $T$ dependence of $\mu_{\alpha}$ but their zero-point fluctuations can cause a considerable isotropic reduction of the overall values.

${ }^{23}$ We have carefully checked that, for the $T$ region of Fig. 1, smaller values of $\hbar \Gamma$ do not change the $T$ dependence but lead only to a scaling $\propto 1 / \Gamma$ of the mobilities.

${ }^{24}$ D. Emin, Phys. Today 6, 34 (1982). 